\title{
Clinical and Radiological Results over the Medium Term of Isolated Acetabular Revision
}

\author{
Nicola Piolanti, Lorenzo Andreani, Paolo Domenico Parchi, \\ Enrico Bonicoli, Francesco Niccolai, and Michele Lisanti
}

\begin{abstract}
1st Orthopedic Division, Department of Translational Research and New Technologies in Medicine, University of Pisa, Via Paradisa 2, 56124 Pisa, Italy
\end{abstract}

Correspondence should be addressed to Nicola Piolanti; nicpio@hotmail.it

Received 31 July 2014; Accepted 14 December 2014; Published 28 December 2014

Academic Editor: Lars Weidenhielm

Copyright (C) 2014 Nicola Piolanti et al. This is an open access article distributed under the Creative Commons Attribution License, which permits unrestricted use, distribution, and reproduction in any medium, provided the original work is properly cited.

\begin{abstract}
Acetabular cup loosening is associated with pain, reduced function, and instability of the implant. If such event happens while the femoral implant is in a satisfactory position and is well fixed to the bone, isolated acetabular revision surgery is indicated. The aim of this single-center retrospective study was to evaluate the clinical and radiological results over the medium term (12-month followup mean 36, max 60) of isolated acetabular revisions surgery using a porous hemispheric revision shell matched with a cemented all-poly cup and large diameter femoral head $(>32)$. 33 patients were enrolled. We collect any relevant data from the clinical board. Routine clinical and radiographic examinations were performed preoperatively; the postoperative follow-up was made at 1,3 , and 6 months and yearly thereafter. At the last available follow-up, we report satisfactory improvement of functional scores in all the patients; 2 patients $(6.1 \%)$ showed thigh pain and only 4 hips (12.11\%) presented mild groin pain; all the femoral components are well fixed and there were no potential or pending rerevisions. With bias due to the follow-up and to the retrospective design of the study, we report clinical, functional, and radiological satisfactory results.
\end{abstract}

\section{Introduction}

The most common reason for failure of total hip arthroplasty (THA) is periprosthetic osteolysis and loosening of hip implants [1]. The rate of osteolysis varies between femoral and acetabular sides, and it is more common on the acetabular side. This is why acetabular cup loosening is the main cause for revision in long-term studies [2]. This loosening usually is associated with pain, reduced function, and instability of the implant.

There are two main problems to solve when an orthopaedic surgeon has to approach acetabular revision. First of all, even with bone loss due to the loosening of the previous implant, obtain primary fixation of the new prosthesis; then reach postoperative implant stability.

This second issue could be more difficult when isolated acetabular revision is performed $[3,4]$.

Effectively in these cases not only does the presence of the stem limit surgical options, but also repeated surgical incision, soft tissue damage, and in some cases extended synovectomy can reduce the stability of the implant [5].
To address this risk, industries and surgeons have developed a variety of surgical hardware and strategies such as jumbo femoral heads [6], constrained acetabular liners [7] and dual-mobility cup [8].

Another way to face these problems is, in order to obtain fixation, the implantation of a shell in the better position allowed by the bone defect; then obtain stability cementing a polyethylene liner in the shell with a partially independent version and verticality.

The present study was conducted to evaluate the clinical and radiological results over the medium term ( $>12$-month follow-up mean 36, max 60) of isolated acetabular revisions surgery using a porous hemispheric revision shell matched with a cemented all-poly cup and large diameter femoral head $(>32)$.

\section{Material and Methods}

This single-center retrospective study was approved by our local ethical committee, and the patients gave written consent 


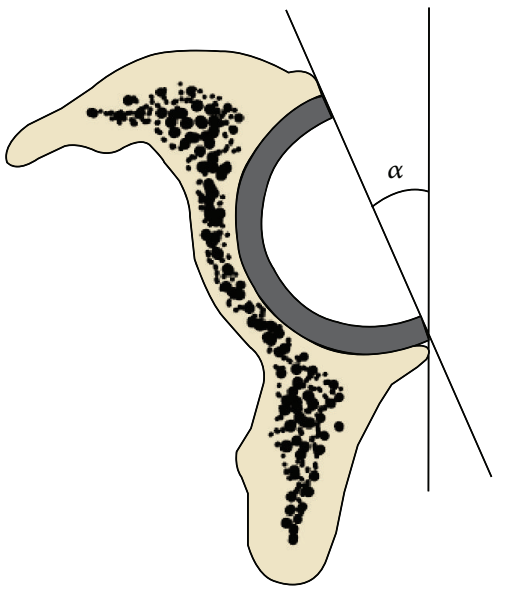

(a)

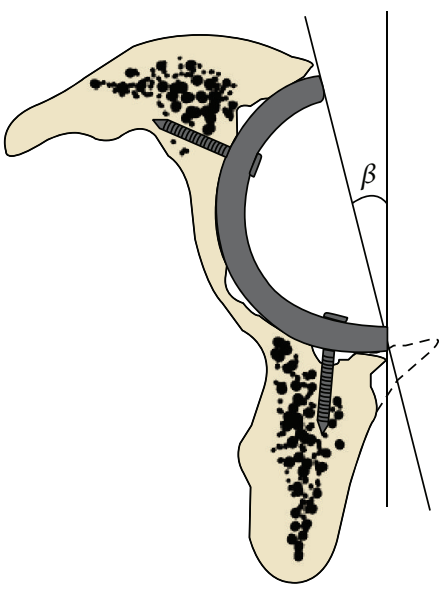

(b)

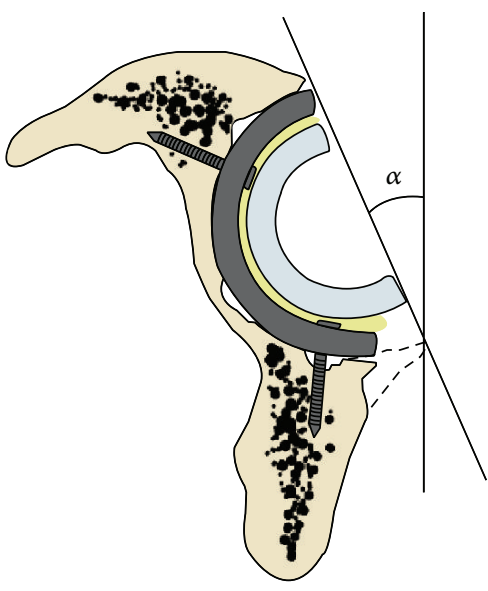

(c)

Figure 1: (a) $\alpha$ angle shows the correct acetabular anteversion reached after primary hip implant. (b) $\beta$ angle obtained after revision hip surgery: the shell was implanted in the better position allowed by the bone defect. (c) During the revision surgery, in order to obtain the stability of the implant, the polyethylene liner was cemented into the shell in order to obtain the correct verticality and anteversion.

to participate. A review of our database between January 2009 and December 2012 for revision hip arthroplasty was done. In this period we performed 86 THA revisions; we selected patients that had isolated acetabular revisions with a porous hemispheric revision shell matched with a cemented all-poly cup and large diameter femoral head (>32).

Information of ages, sex, clinical history, drug treatment, and preoperative and postoperative X-ray studies were collected and recorded. The acetabular defects were classified as described by Paprosky et al. [9].

We included patients with (1) loosening or malpositions of the acetabular components, (2) a well fixed and well positioned femoral stem. We excluded (1) patients with septic loosening, (2) patients that required revision for both components, and (3) patients with monoblock stem. All the patients were operated on in the lateral decubitus position, and the surgical approach was posterolateral. We checked the stability of the stem and then we removed the acetabular cups, liners, and screws. If required before implantation of the revision components pelvic bony defects were grafted with tricalcium phosphate hydroxyapatite and morcelized bone graft. In all the index patients, the Regenerex revision shell (Biomet Warsaw, IN, USA) was implanted. This is a porous titanium construct, with multiple holes to maximize intraoperative screws fixation, designed to accept a cemented all-poly cup. During the revision surgery, the shell was implanted in the better position allowed by the bone defect; then in order to obtain the stability of the implant, the polyethylene liner was cemented into the shell in order to obtain verticality of about 45 degrees and summed of about 35 to 50 degrees of anteversion (stem plus liner) (Figure 1).

At the end of the surgical procedure, we obtained an intraoperative acceptable stability; in our opinion this is defined as $45^{\circ}$ or more of internal rotation at $90^{\circ}$ of flexion and $20^{\circ}$ or more of external rotation in $10^{\circ}$ of hyperextension. The wound was drained in each case.
In the postoperative period all the patients performed thromboembolic prophylaxis, with low molecular weight heparin; we did not use nonsteroidal anti-inflammatory drugs to prevent heterotopic periprosthetic ossification.

Partial weight bearing was allowed since the first postoperative week in the majority of patients; in some due to poor patient's bone stock we delayed it to the first radiological and clinical follow-up (six weeks) and gradually advanced it to full weight bearing. No postoperative bracing was used.

Routine clinical and radiographic examinations were performed preoperatively; the postoperative follow-up was made at 1,3 , and 6 months and yearly thereafter. Patients were scored as routinely in our practice preoperatively with the Harris Hip Score (HHS) and the Western Ontario and McMaster Universities Osteoarthritis Index (WOMAC) [10, 11]; the same scores were used during the follow-up. As routinely during clinical evaluation, we collect and report data about any relevant adverse event that occurred; in addition we also investigate if there is thigh or groin pain, any subjective perception of instability, and subsequent apprehension.

Radiographs were evaluated by two of the senior authors (Enrico Bonicoli and Paolo Domenico Parchi), with consensus attained for reporting of all measurements. The radiographic follow-up was performed in order to evaluate the position of the implant and to search for any signs of osseointegration or loosening of the components.

The de Lee and Charnley classification [12] was used; radiolucent lines were considered present if they were greater than $1 \mathrm{~mm}$ at their maximum width and involved any two adjacent sectors of the cup surface [13]. A horizontal or vertical change in position of at least $3 \mathrm{~mm}$, or a change in abduction angle of at least $5^{\circ}$, was considered migration [14]. According to Brooker et al., on the last available X-ray, we also evaluated heterotopic ossification [15].

The Wilcoxon signed-rank test was used to compare preoperative and postoperative hip scores. 

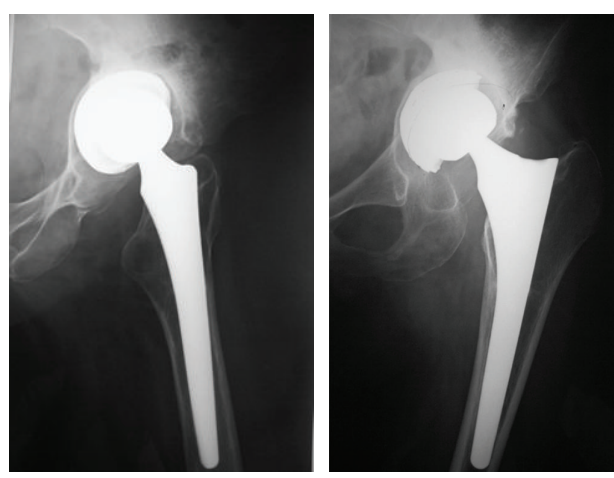

(a)

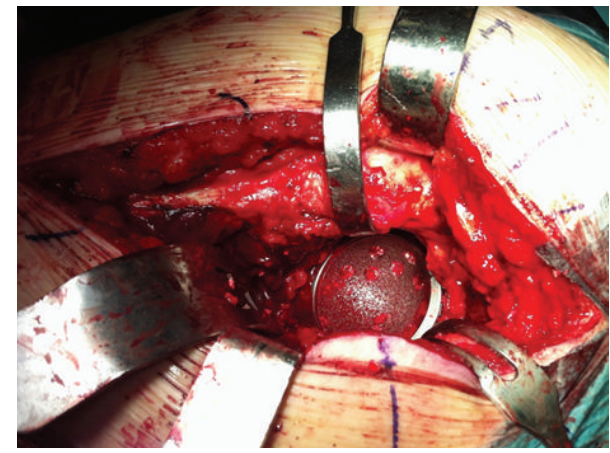

(c)

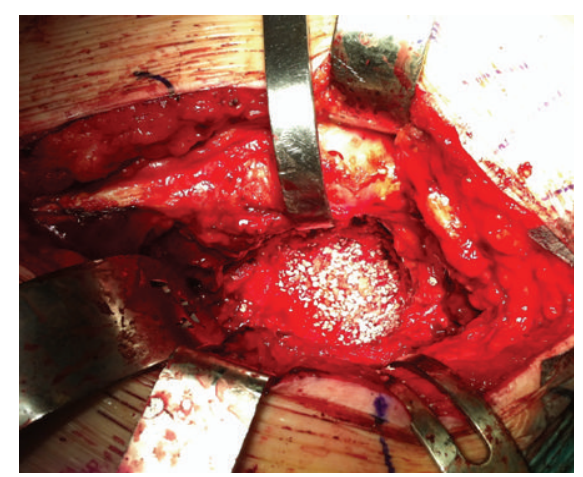

(b)

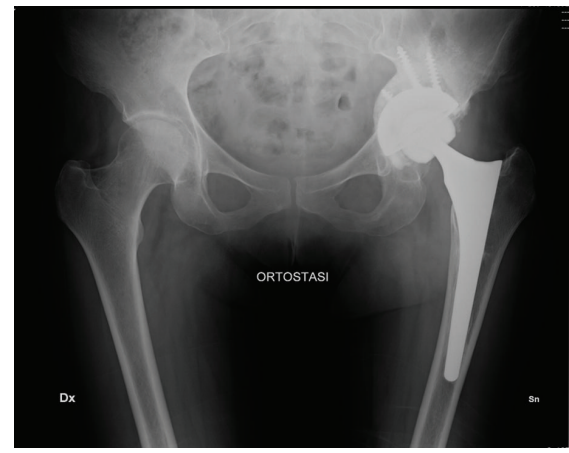

(d)

Figure 2: (a) Loosening of an acetabular press fit cup, stable stem. (b) Intraoperative picture showed bone defect grafted with tricalcium phosphate hydroxyapatite and morcelized bone graft. (c) Regenerex revision shell in place. (d) X-ray at the last available follow-up showing acetabular integration.

\section{Results}

33 patients were enrolled for this study. There were 23 women $(69,7 \%)$ and 10 men $(30,3 \%)$; the mean age at the revisions was 67 years (range $40-81$ years); 17 were left $(51,5 \%)$ and $16(48,5 \%)$ were right. The mean clinical follow-up was 36 months (minimum 13 months, maximum 60 months); one died from unrelated illnesses; 2 patients were lost to followup.

The preoperative diagnoses were 26 with aseptic loosening $(78,79 \%)$, 3 with implant instability $(9,1 \%)$, and 4 revisions due to metallosis $(12,11 \%)$.

We treated 9 with type 1 defect of the Paprosky classification [9] (27\%), 5 with type $2 \mathrm{a}(15 \%), 7$ with type $2 \mathrm{~b}$ (21\%), 5 with type $2 \mathrm{c}(15 \%)$, and 7 with type $3 \mathrm{a}(21 \%)$. Cup trend was size 56, max size 68 and min size 54 . In 13 patients, acetabular bone defects were grafted using tricalcium phosphate hydroxyapatite and morcelized bone graft (Figure 2); in 2 patients we used titanium augmentation. The mean number of screws used to secure the revision shells was five (from 3 to 7). The size of the femoral head was 32 (21 hips) and $36 \mathrm{~mm}$ (12 hips). See Table 1 for more details.

Early complications (during the in-patient stay) occurred in 4 of the 33 patients $(12,11 \%)$. These included a superficial wound infection in two, deep-vein thrombosis in one, and postoperative early dislocation in one.
The postoperative early dislocation happened during a possible wrong movement of the patient from the operative room to the ward in a patient under spinal anesthesia. We reduced the dislocation with external manoeuvres and this event also at the last follow-up never happened again.

The mean WOMAC score in 25 patients improved significantly from 52.1 preoperatively to 62.27 postoperatively $(P=0.008)$. The mean Harris Hip Score improved from 59 points (range 43-74) preoperatively to 88 points (range 6792) $(P=0.002)$. At the final follow-up, 2 patients $(6,1 \%)$ showed thigh pain and only 4 hips (12.11\%) presented mild groin pain; all the femoral components are well fixed and there were no potential or pending rerevisions.

With regard to the radiological evaluation, according to de Lee and Charnley [12], at the initial follow-up, we found 7 (21\%) of the Regenerex implants with radiolucent lines bigger than $1 \mathrm{~mm}$ at their maximum width and which involved one or two adjacent sectors of the cup surface. In the last examination, five of the seven cases with radiolucent lines remaining still visible on the X-rays and seem not to be evolutive. In agreement with Schmalzried and Harris [16] and Petersen et al. [17], we attribute the filling of the gap to the formation of new bone (Figure 3).

In 3 patients osteolysis around the screws was noted, without any change of the cup orientation and without evident evolution. No change of position was found. Even in 


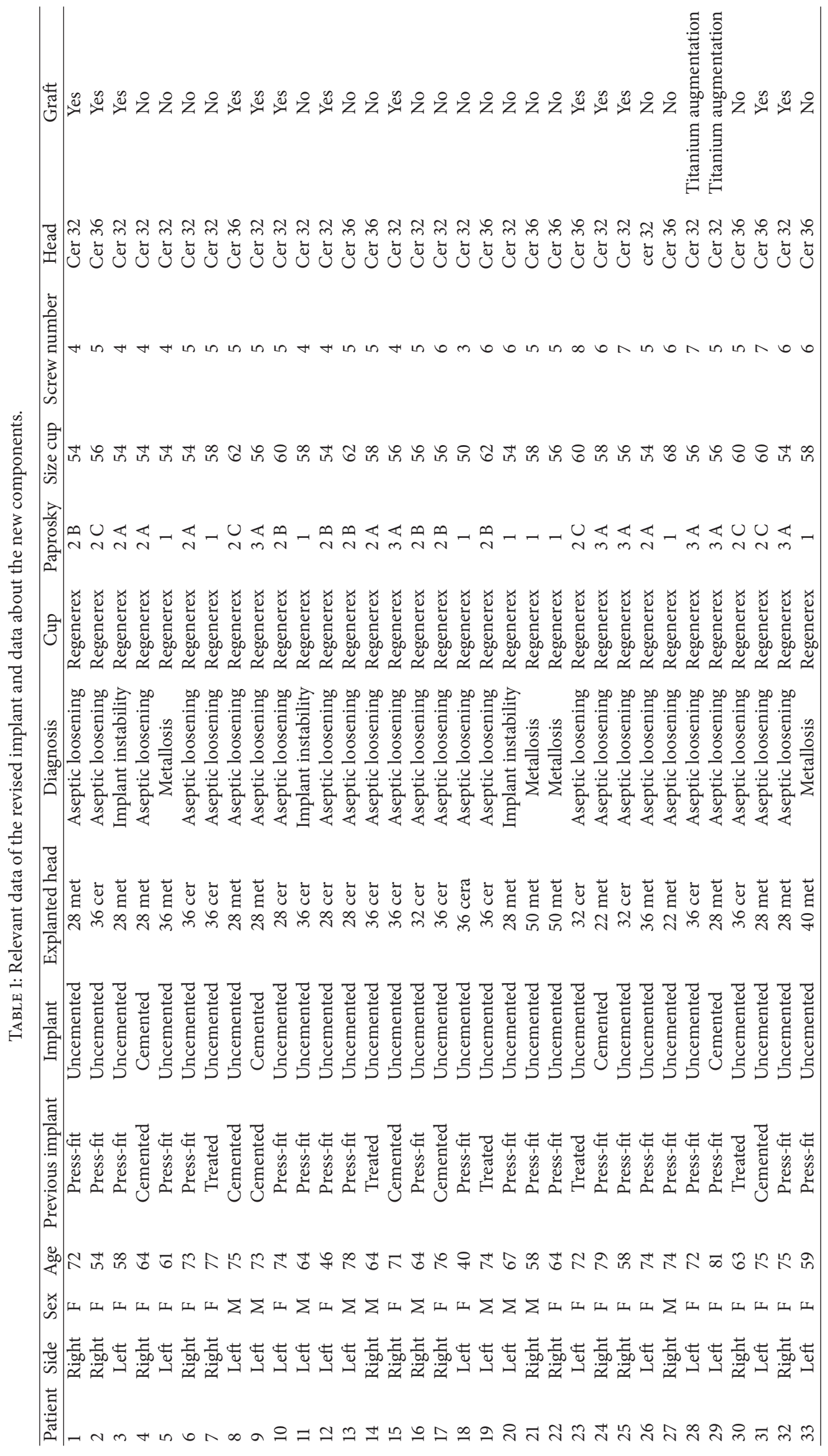




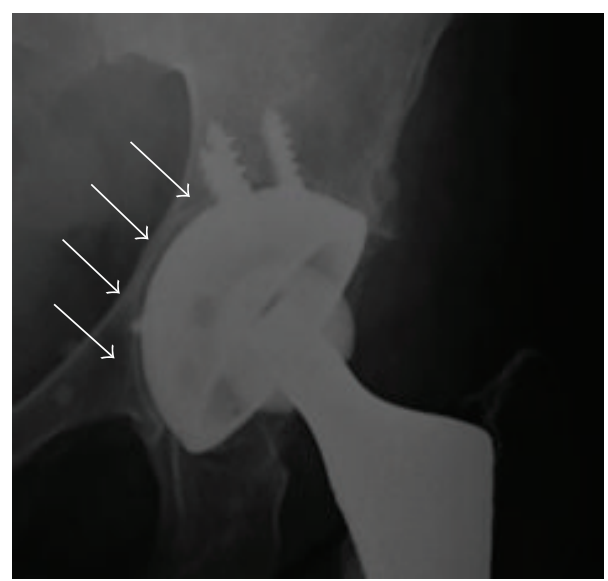

(a)

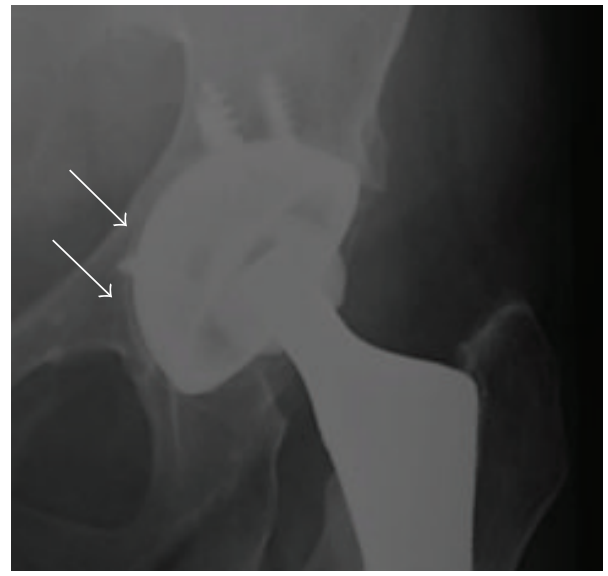

(c)

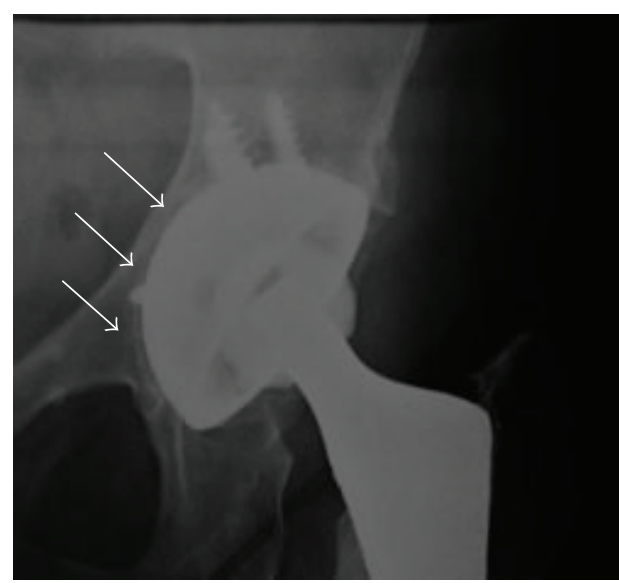

(b)

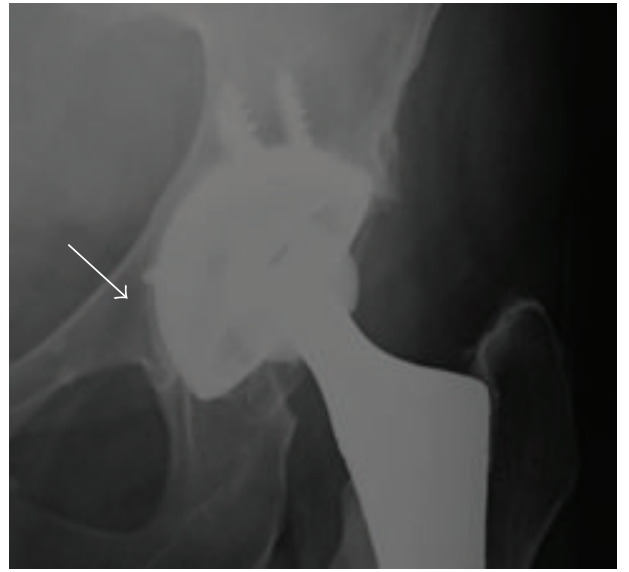

(d)

Figure 3: (a) Postoperative radiographs showing radiolucent lines which involved two adjacent sectors of the cup surface. ((b), (c), and (d)) Progressive osseointegration during 3, 6, and 12 months' follow-up.

our series it is not possible to establish the influence of bone grafting for the final stabilization of the implant; when it was used, good bone osseointegration was seen.

Heterotopic ossification type 1-2 of Brooker classification [14] was found in 3 patients.

We do not report major $(>2 \mathrm{~cm}$ ) leg length discrepancy. At the most recent follow-up no one patient had experienced a further dislocation.

\section{Discussion}

In this study we evaluate the clinical and radiological results over the medium term of isolated acetabular revision with a porous hemispheric revision shell matched with a cemented all-poly cup and large diameter femoral head ( $>32)$.

Besides the lack of a control group there are some limitations in this study, such as the short follow-up of same patients and the retrospective design.

However we think that our study can improve the literature's knowledge about this issue also because as reported in the Australian registry, the revision of the acetabular component is the most common cause of repeated surgery in total hip replacement [18]. Isolated acetabular revision is indicated when an acetabular implant is associated with pain, reduced function, instability, or loosening, while the femoral implant is in satisfactory position and is well fixed to bone. The benefits of leaving the femoral component in place include reduced operating time, less blood loss, and preservation of bone stock [19]. Doubtless the presence of the femoral implant limits the surgical exposure, making access to the acetabulum and treatment of bone defects challenging and increasing the risk of postoperative dislocation.

The author's choice to face the problem of achieving fixation of the implant is to choose porous materials such as Regenerex during revision surgery. The advantage of these materials is not only to provide a primary stability due to scratch fit but also to allow long-term implant stability related to bone ingrowth (osteoconductivity) [20], which is important especially in revision surgery when bone quality is poor [21, 22]. Another advantage of such technique is to implant the shell in the better position allowed by the bone defect reaching the better possible primary stability and then cementing the polyethylene liner with version and verticality partially independent (Figure 2). 
In this way it is possible to obtain the correct geometry of the revised implant (verticality of about 45 degrees and summed of about $35^{\circ}$ to 50 degrees of anteversion, stem plus liner) issue that is important to reduce the risk of dislocation which has been associated with hip revision surgery and rises in isolated acetabular revision.

The author's opinion supported by biomechanical tests and several clinical series with short-term follow-up of liner cementation is that such technique is sure. The cemented polyethylene liner was found to have an initial fixation strength exceeding that of the conventional locking mechanism if 2 and $4 \mathrm{~mm}$ thick cement mantles were built up around the liner [18]. It is undisputed that large femoral heads improve stability of the hip implant by increasing the excursion before dislocation can occur [23]. The literature does not clarify whether any preoperative variables influence pain relief or functional scores [24]; however we report satisfactory improvement of functional scores (HHS improves from 59 to 88 and WOMAC from 52,1 to 62,27 ) with only 4 patients $(12,11 \%)$ complaining of mild groin pain and $2(6,1 \%)$ patients with thigh pain. We do not report major $(>2 \mathrm{~cm})$ leg length discrepancy. The only one dislocation (3\%) is in-line or even better compared with the results reported from other authors [5-7, 24]; such dislocation appears to be correlated to a wrong patient transfer from the operative to the to the ward matched with spinal anesthesia.

Schneider et al. [5] had 10 cases of dislocation (10.4\%) in a series of 96 revisions with a reconstruction cage and a cemented dual-mobility cup. Della Valle et al. [7] in their series of 55 cases at a mean follow-up of 3,6 years reported a dislocation rate of $16 \%$. Lawless et al. [24] reported a dislocation rate of $0 \%$ at 6,4 years after revision but with a 7,3\% of reoperation due to aseptic loosening. With bias due to the follow-up and to the retrospective design of the study, in our series complications were low and we did not report reoperations, not only for the acetabular component but also for the stem; such results compare favorably with previous report $[8,25]$. In our opinion, selective acetabular revision with a porous hemispheric revision shell matched with a cemented all-poly cup and large diameter femoral head is a reliable alternative with excellent clinical success over the medium term. Precise check of the preoperative X-rays and a careful evaluation of the intraoperative stem stability are mandatory for such results. Nevertheless more detailed and scrupulous evaluation with long-term prospective studies is needed.

\section{Disclosure}

This study comply with the current laws of the country in which it was done (Italy).

\section{Conflict of Interests}

The authors declare that there is no conflict of interests regarding the publication of this paper.

\section{Acknowledgment}

The authors thank Alessia Diaco for graphical support.

\section{References}

[1] A. Rosenberg, "Revision total hip arthroplasty: indications and contra-indications," in Advanced Hip Reconstruction, J. R. Lieberman, J. Daniel, and D. Berry, Eds., pp. 281-283, AAOS, Rosemont, Ill, USA, 2005.

[2] P. Herberts, L. Ahnfelt, H. Malchau, C. Stromberg, and G. B. J. Andersson, "Multicenter clinical trials and their value in assessing total joint arthroplasty," Clinical Orthopaedics and Related Research, no. 249, pp. 48-55, 1989.

[3] K. Fukui, A. Kaneuji, T. Sugimori, T. Ichiseki, K. Kitamura, and T. Matsumoto, "Should the well-fixed, uncemented femoral components be revised during isolated acetabular revision?" Archives of Orthopaedic and Trauma Surgery, vol. 131, no. 4, pp. 481-485, 2011.

[4] J. T. Moskal, F. H. Shen, and T. E. Brown, "The fate of stable femoral components retained during isolated acetabular revision: a six-to-twelve-year follow-up study," Journal of Bone and Joint Surgery-Series A, vol. 84, no. 2, pp. 250-255, 2002.

[5] L. Schneider, R. Philippot, B. Boyer, and F. Farizon, "Revision total hip arthroplasty using a reconstruction cage device and a cemented dual mobility cup," Orthopaedics and Traumatology: Surgery and Research, vol. 97, no. 8, pp. 807-813, 2011.

[6] P. E. Beaulé, T. P. Schmalzried, P. Udomkiat, and H. C. Amstutz, "Jumbo femoral head for the treatment of recurrent dislocation following total hip replacement," The Journal of Bone \& Joint Surgery A, vol. 84, no. 2, pp. 256-263, 2002.

[7] C. J. Della Valle, D. Chang, S. Sporer, R. A. Berger, A. G. Rosenberg, and W. G. Paprosky, "High failure rate of a constrained acetabular liner in revision total hip arthroplasty," Journal of Arthroplasty, vol. 20, supplement 3, pp. 103-107, 2005.

[8] R. Civinini, C. Carulli, F. Matassi, L. Nistri, and M. Innocenti, "A dual-mobility cup reduces risk of dislocation in isolated acetabular revisions," Clinical Orthopaedics and Related Research, vol. 470, no. 12, pp. 3542-3548, 2012.

[9] W. G. Paprosky, P. G. Perona, and J. M. Lawrence, "Acetabular defect classification and surgical reconstruction in revision arthroplasty: a 6-year follow-up evaluation," Journal of Arthroplasty, vol. 9, no. 1, pp. 33-44, 1994.

[10] W. H. Harris, "Traumatic arthritis of the hip after dislocation and acetabular fractures: treatment by mold arthroplasty. An end-result study using a new method of result evaluation," Journal of Bone and Joint Surgery-Series A, vol. 51, no. 4, pp. 737-755, 1969.

[11] N. Bellamy, W. W. Buchanan, C. H. Goldsmith, J. Campbell, and L. W. Stitt, "Validation study of WOMAC: a health status instrument for measuring clinically important patient relevant outcomes to antirheumatic drug therapy in patients with osteoarthritis of the hip or knee," The Journal of Rheumatology, vol. 15, no. 12, pp. 1833-1840, 1988.

[12] J. G. de Lee and J. Charnley, "Radiological demarcation of cemented sockets in total hip replacement," Clinical Orthopaedics and Related Research, vol. 121, pp. 20-32, 1976.

[13] M. S. Moore, J. P. McAuley, A. M. Young, and C. A. Engh Sr., "Radiographic signs of osseointegration in porous-coated acetabular components," Clinical Orthopaedics and Related Research, no. 444, pp. 176-183, 2006. 
[14] P. Massin, L. Schmidt, and C. A. Engh, "Evaluation of cementless acetabular component migration: an experimental study," Journal of Arthroplasty, vol. 4, no. 3, pp. 245-251, 1989.

[15] A. F. Brooker, J. W. Bowerman, R. A. Robinson, and L. H. Riley Jr., "Ectopic ossification following total hip replacement: incidence and a method of classification," The Journal of Bone \& Joint Surgery A, vol. 55, no. 8, pp. 1629-1632, 1973.

[16] T. P. Schmalzried and W. H. Harris, "The Harris-Galante porous-coated acetabular component with screw fixation. Radiographic analysis of eighty-three primary hip replacements at a minimum of five years," Journal of Bone and Joint Surgery, vol. 74, no. 8, pp. 1130-1139, 1992.

[17] M. B. Petersen, I. H. Poulsen, J. Thomsen, and S. Solgaard, “The hemispherical Harris-Galante acetabular cup, inserted without cement: the results of an eight to eleven-year follow-up of one hundred and sixty-eight hips," Journal of Bone and Joint Surgery-Series A, vol. 81, no. 2, pp. 219-224, 1999.

[18] C. He, J.-M. Feng, Q.-M. Yang, Y. Wang, and Z.-H. Liu, "Results of selective hip arthroplasty revision in isolated acetabular failure," Journal of Surgical Research, vol. 164, no. 2, pp. 228-233, 2010.

[19] C. J. Kershaw, R. M. Atkins, C. A. F. Dodd, and C. J. K. Bulstrode, "Revision total hip arthroplasty for aseptic failure: a review of 276 cases," Journal of Bone and Joint Surgery B, vol. 73, no. 4, pp. 564-568, 1991.

[20] J. Szypula and J. Kędziora, "The use of titanium sponge in hip revision replacement prosthesis-preliminary report," Polski Merkuriusz Lekarski, vol. 27, no. 160, pp. 315-317, 2009.

[21] R. M. Pilliar, H. U. Cameron, and I. Macnab, "Porous surface layered prosthetic devices," Bio-Medical Engineering, vol. 10, no. 4, pp. 126-131, 1975.

[22] E. Bonicoli, N. Piolanti, L. Andreani, P. Parchi, and M. Lisanti, "Preliminary report with the Regenerex revision shell: clinical, functional, and radiologic evaluations with a mean follow-up of 25 months," European Orthopaedics and Traumatology, vol. 4, no. 1, pp. 9-14, 2013.

[23] S. Byström, B. Espehaug, O. Furnes, and L. I. Havelin, "Femoral head size is a risk factor for total hip luxation: a study of 42,987 primary hip arthroplasties from the Norwegian Arthroplasty Register," Acta Orthopaedica Scandinavica, vol. 74, no. 5, pp. 514-524, 2003.

[24] B. M. Lawless, W. L. Healy, S. Sharma, and R. Iorio, "Outcomes of isolated acetabular revision," Clinical Orthopaedics and Related Research, vol. 468, no. 2, pp. 472-479, 2010.

[25] A. Moličnik, M. Hanc, G. Rečnik, Z. Krajnc, M. Rupreht, and S. K. Fokter, "Porous tantalum shells and augments for acetabular cup revisions," European Journal of Orthopaedic Surgery \& Traumatology, vol. 24, no. 6, pp. 911-917, 2014. 


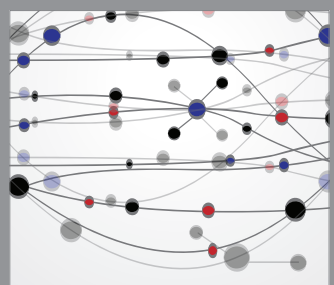

The Scientific World Journal
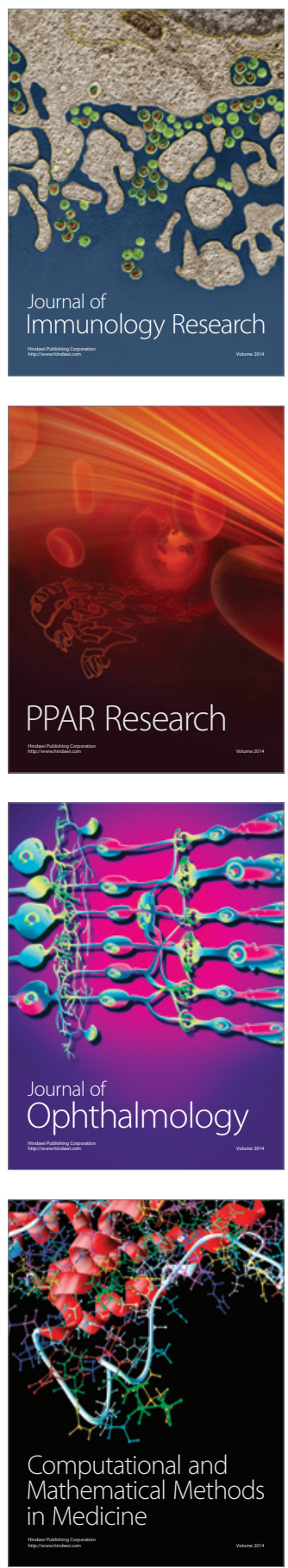

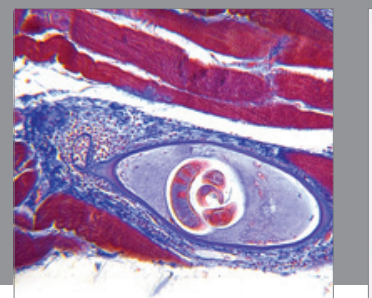

Gastroenterology

Research and Practice
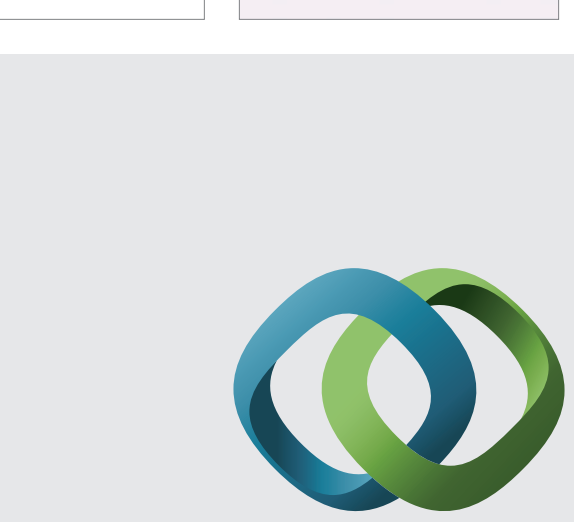

\section{Hindawi}

Submit your manuscripts at

http://www.hindawi.com
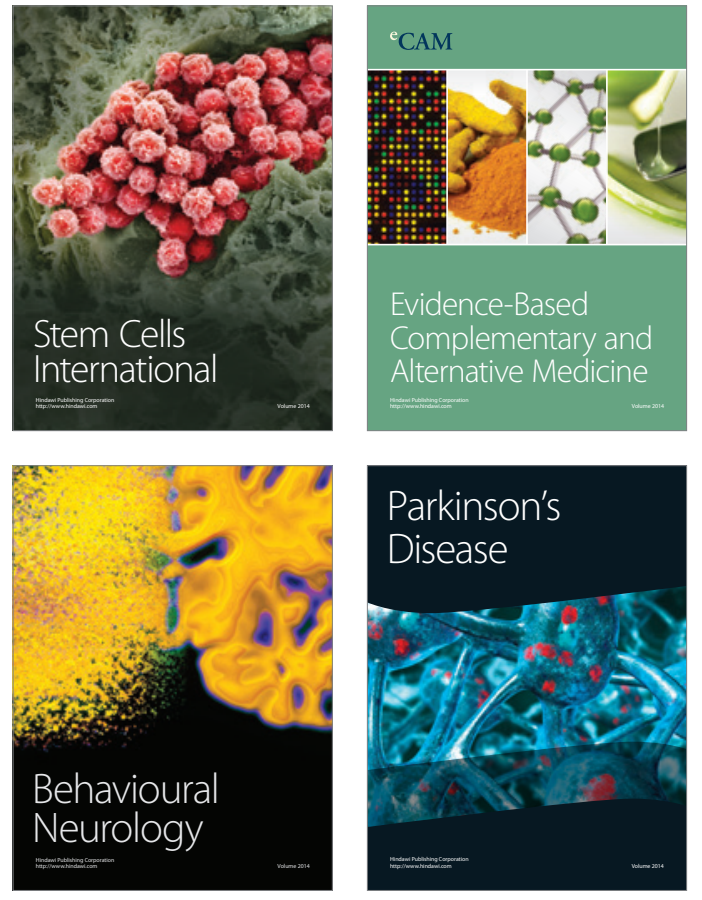
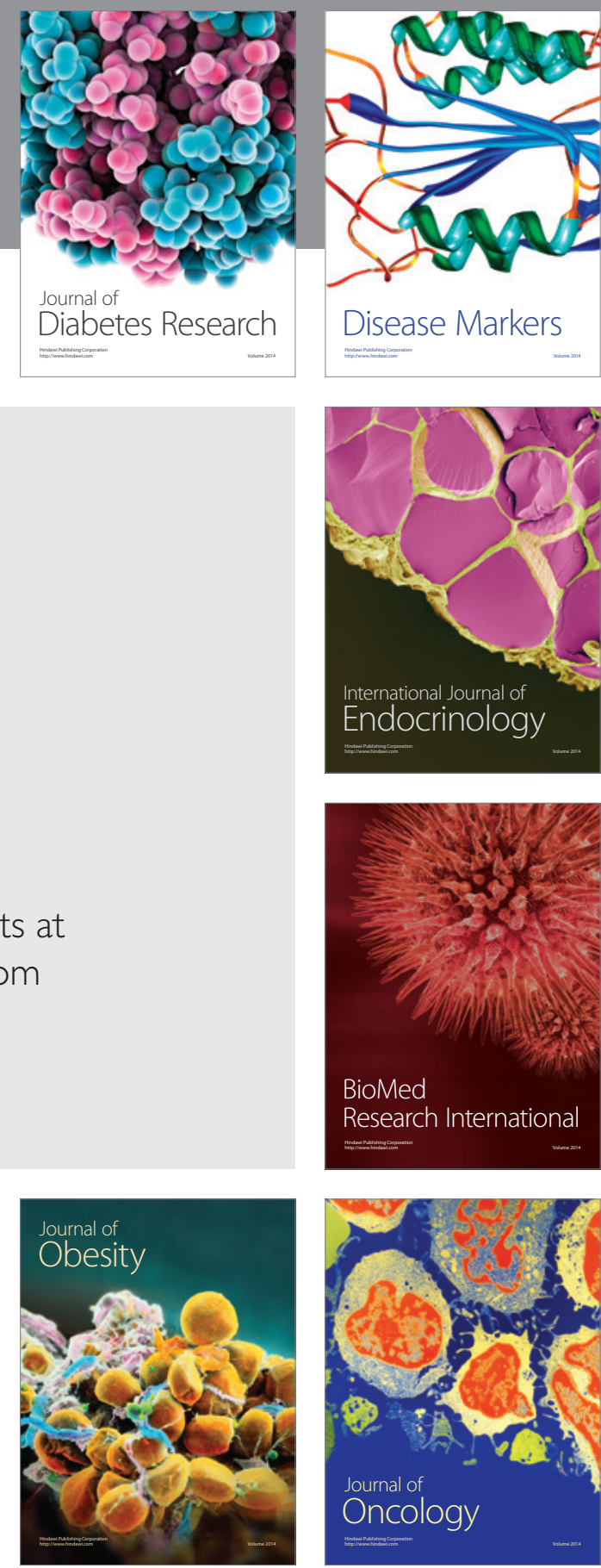

Disease Markers
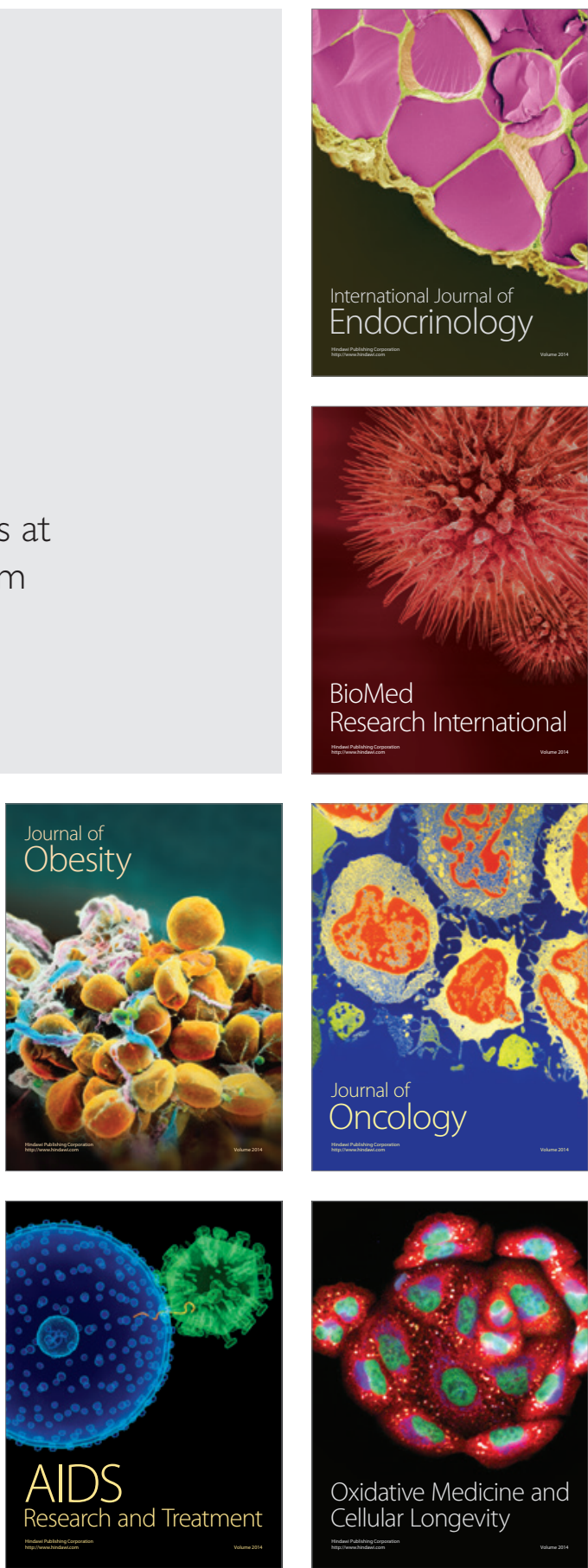